\title{
GPU 加速的表意式体模型线绘制算法
}

\author{
张龙 ${ }^{(1)} ，$ 王毅刚 ${ }^{(1)}$ ，吴向阳 ${ }^{(2)}$ \\ (1)杭州电子科技大学数字媒体与艺术设计学院, 杭州 310018 \\ (2)杭州电子科技大学计算机学院, 杭州 310018 \\ * 通信作者. E-mail: hnex.zhang@gmail.com
}

收稿日期: 2014-07-20; 接受日期: 2014-09-29

国家自然科学基金 (批准号: 60903085, 61170150) 和浙江省自然科学基金 (批准号: LY13F020048, Z1091077) 资助项目

\begin{abstract}
摘要 现有的体模型线绘制算法采用跟踪随机种子的方式得到特征线, 存在严重的时空连续性问题. 本文提出一种 GPU 加速的体模型线绘制算法, 能够在每一帧抽取全部的特征线, 从而彻底解决绘制 中的不连续现象. 我们首先提出一种并行的特征线抽取方法, 在 GPU 的几何处理器中计算每个立方 体单元中的特征线片段. 为了解决可见性判定问题, 我们采用 splatting 的方式生成深度图, 取代已有 方法中的光线投射方法, 获得了明显的性能提升. 采用自适应的深度偏移获得满意的可见性检测结 果. 为了将大量的体数据存储到高速显存中, 我们通过剔除无关数据和压缩编码的方式, 在不影响结 果的前提下显著地减少了存储和带宽消耗. 实验结果表明, 本文算法能够生成时空连续的线绘制结 果, 并且绘制速度比已有 $\mathrm{CPU}$ 算法提高一个数量级.
\end{abstract}

关键词 绘制 体绘制 特征提取 图解 加速

\section{1 引言}

线绘制算法是计算机图形学中的一个重要研究课题, 其主要研究内容是如何基于给定的三维模型 自动或半自动地生成相应的线条画, 从而表现给定模型的几何形状. 线绘制的研究具有多方面的意义, 其中一个最明显的作用是通过生成线条画可以清晰地揭示模型的形状特征. 例如: 文献 [1] 使用线绘 制的技术表现了普通照片中不易被察觉的形状特征; 文献 [2] 将线绘制的技术成功地应用到考古学中, 表现古文物的形状特征, 获得了与文物工作者手工描绘相近的结果.

在过去的十年中, 线绘制技术获得了广泛的关注. 许多学者围绕 “在哪里画线” 这一问题, 提出了 各种各样的特征线定义方法, 从不同的角度描述三维模型的形状特征 (如文献 [3 5] 等). 另外有许多 方法采用图像滤波的技术在图像空间中生成线绘制结果 ${ }^{[6,7]}$. 此外, 关于线绘制的加速技术 ${ }^{[8]}$ 以及对 于模型几何噪声与拓扑噪声的鲁棒性等问题 [9] 也提出了很好的解决方案.

尽管线绘制算法获得了充分的研究, 但是绝大多数的研究工作都是针对于多边形网格模型, 只有 极少数的方法可以应用于体模型. 而事实上, 线绘制技术对于体模型的表意式绘制具有非常重要的意 义. 特别的, 由于特征线的分布通常比较稀疏, 因此可以将多层表面的线绘制结果叠加在一起, 自然地 表现体模型的内部空间结构. 
与网格模型相比, 体模型的数据量要大得多. 例如, 一个中等尺寸的 $256^{3}$ 分辨率的体模型就具有 1600 多万个体素, 远大于一般网格模型的顶点数量. 因此, 体模型的线绘制算法必须要考虑大数据量 带来的绘制效率和存储消耗的问题. 为了提高绘制效率, 现有的体模型线绘制算法 $[10,11]$ 采用 “随机 种子跟踪” 的方法来加速计算. 首先随机产生一些体素, 测试它们是否与某条特征线相交. 将相交的体 素作为种子点, 沿着种子点的相邻体素进行跟踪直至抽取出整根特征线. 实验结果表明, 随机种子跟 踪能够显著地提高线绘制效率 (大致能将算法复杂度从 $O\left(n^{3}\right)$ 降为 $O(n)$ ). 但是, 这一方法也带来很 多的问题. 首先, 使用随机种子跟踪的方法无法确保生成的线绘制结果是完整的, 或者说, 无法确定是 否抽取出所有的特征线. 其次, 由于每一帧随机选择的种子点不同, 造成相邻帧生成的特征线不一致, 从而产生较为严重的闪炼现象. 现有的解决方法是在绘制当前帧时首先测试上一帧的所有种子点, 然 后再额外添加若干新的种子点. 这一方法能够在一定程度上缓解闪伢的问题, 但是由于新种子点的引 入, 闪炼的现象仍然存在.

本文提出一种基于 GPU 实现的体模型线绘制算法. 已有的随机种子跟踪算法是一种串行的算法, 难以在 GPU 中实现, 并且如前所述会导致时空连续性的问题, 为此我们舍弃了这一方法, 而是通过对 体数据进行桶分类的方法降低计算复杂度. 为了在 GPU 中高效实现, 我们对特征线的抽取进行了一 定的简化和近似. 实验结果表明, 我们的方法生成的结果与完整结果几乎没有视觉上的差别, 但是所 需时间大大减少. 此外, 为了将有用的体数据加载到高速的 GPU 显存, 我们实现了高效的数据剔除和 压缩算法. 具体而言, 我们的技术贡献包括以下三个方面:

(1) 提出了一种并行的特征线抽取算法 (4.1 小节), 可以高效地通过 GPU 中的几何处理器 (geometry shader) 并行计算每个立方体单元中的特征线片段, 计算结果与正确结果几乎没有差别, 而计算速 度提高近 20 倍.

(2) 提出了一种基于 splatting 的深度图生成与可见性判定方法 (4.2 小节), 区分可见与不可见表 面的特征线. 通过自适应的深度偏移提高可见性判定的准确率.

(3) 实现高效的数据剔除与压缩算法 (4.3 小节), 在不损失绘制精度的前提下将数据量减少为原有 数据的 $10 \%$ 至 $30 \%$, 显著地减少了数据传输的带宽, 提高了绘制效率.

\section{2 相关工作}

\section{1 特征线定义}

如何定义模型的特征, 即 “在哪里画线” 是线绘制算法需要解决的最根本的问题. 在图形学中, 通常 采用数字几何处理的方法, 将特征线定义为模型表面上满足某种几何约束的点的集合. 例如, 最经典的 侧影轮廓线, 是法向与视线方向垂直, 即法向与视线方向点积为 0 的点集合. Suggestive contour $^{[3]}$ 是对轮廓线的自然扩展, 定义为法向与视线方向点积具有局部最小值的点集合. 凹凸线 (ridge and valleys) ${ }^{[4]}$ 是曲率局部最大的点集合. Apparent ridges ${ }^{[5]}$ 是考虑投影影响的 “视点相关的曲率” 局 部最大的点. 除此之外, 优秀的特征线定义方法还有 PELs ${ }^{[12]}$, 拉普拉斯 (Laplace) 线 ${ }^{[11]}$, Demarcating

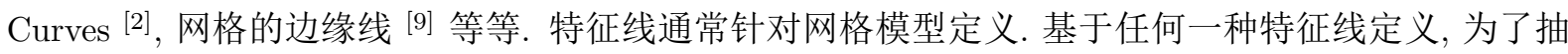
取特征线的几何, 需要对模型的每个顶点分别计算相应的几何约束, 然后逐面片计算其中的特征线片 段. 为了获得实时的效率, 通常采用离散微分几何的方法, 基于顶点的一环或二环邻域计算相应的几 何约束.

本文提出的是一个统一的体模型线绘制框架, 可以无需抽取等值面而直接生成线绘制结果. 从理 
论上讲, 任何一种特征线定义均可集成到我们的算法框架中.

\section{2 体模型的线绘制算法}

为了生成体模型的线绘制结果, 一种做法是首先采用移动立方体等方法抽取等值面, 然后对等值 面应用网格模型的线绘制算法 (例如文献 [12]) . 但是, 使用移动立方体抽取等值面 (特别是重建等 值面的拓扑结构) 是一个比较耗时的操作. 再者, 生成的等值面通常网格质量很差, 在不经过光顺处 理的情况下难以生成理想的线绘制结果. 现有的实时体模型线绘制算法 ${ }^{[10,11]}$ 都是基于 “移动线段 法” (marching lines) ${ }^{[13]}$, 直接在体中抽取特征线, 而不需要预先抽取等值面. 与移动立方体类似, 移动 线段法对体数据的每个立方体单元分别进行处理. 根据立方体的 8 个顶点 (体素) 的值, 通过若干预 计算的查找表确定该立法体中是否存在特征线以及定位特征线顶点的位置. 考虑到特征线的存在通常 非常稀疏, 绝大多数的立法体中没有特征线, 已有算法 ${ }^{[10,11]}$ 采用随机种子跟踪的方式以提高效率. 但 是, 如前所述, 这一方法在获得加速的同时也带来严重的时空连续性问题.

\subsection{GPU 加速的线绘制算法}

许多特征线的定义是视点相关的, 需要在每一帧重新抽取特征线. 为了提高线绘制的实时效率, 一 种常用的手段是采用 GPU 加速计算.

文献 $[8,14]$ 分别实现了 GPU 加速的 suggestive contour 和光极线 (PELs) 绘制. 文献 [15] 提出一 系列的算法, 通过 GPU 加速, 能够实时绘制出具有高光材质的铅笔画效果. 文献 [16] 提出一种使用 GPU 实时计算动态模型表面曲率的方法, 可以应用于生成多种线绘制效果. 以上方法均针对网格模型 设计, 并不能直接用于体模型的线绘制. 文献 [17] 将方法 [16] 进一步扩展到计算体模型等值面的曲率. 尽管目前还没有实现, 我们认为该方法可以集成到本文算法框架中, 通过实时计算等值面曲率从而实 现凹凸线和 suggestive contour 的体模型线绘制效果. 文献 [18] 提出了一种基于多次 splatting 的线绘 制方法. 该方法可以同时应用于网格模型与体模型, 并且生成的线绘制效果很好, 但是它是一种图像 空间的算法, 只能生成线绘制的效果, 而本文提出的是一种物体空间的线绘制算法框架, 可以抽取三 维空间的特征线几何.

\section{3 算法概要}

本文算法的输入是一个规则体模型 $\mathcal{V}$. 体模型 $\mathcal{V}$ 由一个三维阵列的体素构成, 每个体素 $(x, y, z)$ 具有一个标量值 $f(x, y, z)$. 算法输出是体模型 $\mathcal{V}$ 的特征线, 定义为两个等值面 $S_{f}$ 与 $S_{g}$ 的交线. 其中, $S_{f}=\left\{(x, y, z) \mid f(x, y, z)=f_{0}\right\}$ 是体素值等于 $f_{0}$ 的等值面, 值 $f_{0}$ 由用户指定, 可以实时调节, 从而生 成不同等值面的特征线; $S_{g}=\{(x, y, z) \mid g(x, y, z)=0\}$ 是满足某个特征函数 $g$ 等于 0 的等值面. 其中, 特征函数 $g$ 根据不同的特征线有不同的定义方法. 例如, 轮廓线的特征函数定义为 $g(x, y, z)=\boldsymbol{n} \cdot \boldsymbol{v}$, $\boldsymbol{n}$ 与 $\boldsymbol{v}$ 分别表示各体素的法向与视线方向.

如图 1 所示, 算法总体分为预处理和实时绘制两个阶段. 在预处理阶段, 完成体数据的分析, 剔除 无用数据; 对有用数据完成桶分类; 计算各个体素的法向等信息, 将数据通过压缩编码保存在 GPU 显 存中. 在实时绘制阶段, 首先采用 splatting 的方法生成可见面的深度图; 然后对每个由相邻 8 个体素 构成的立方体单元, 抽取可能存在的特征线段; 最后基于深度图判定各个特征线段的可见性, 输出线 绘制结果. 


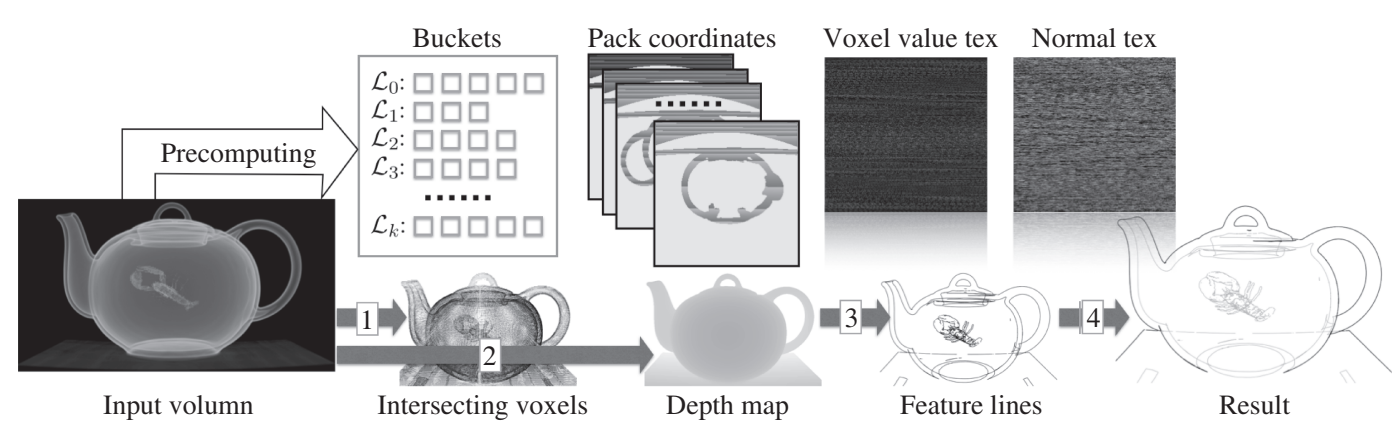

图 1 本文算法流程. 在预处理阶段, 对体数据进行桶分类, 将各种数据通过压缩编码保存为多个纹理. 实时绘制分 为 4 步: (1) 找出与当前等值面相交体素; (2) 通过 splatting 生成深度图; (3) 逐立方体单元抽取特征线片段; (4) 可见性判定

Figure 1 The algorithm pipeline. In the preprocessing stage, we divide the voxels into a set of buckets, and we compress and encode the data in a few textures. The real-time rendering stage consists of four steps: (1) Collect all voxels intersecting the current isosurface; (2) render the depth map using point splatting; (3) extract the feature line segment in each cell; (4) determine the visibility for each feature line segment

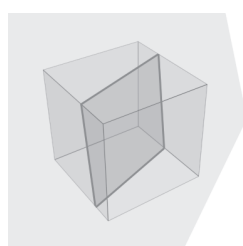

(a)

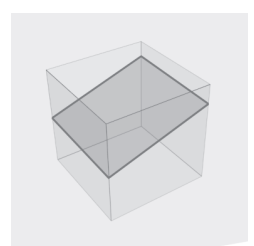

(b)

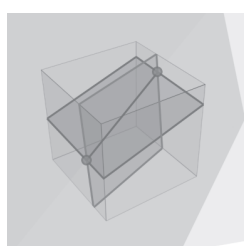

(c)

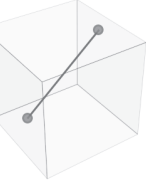

(d)

图 2 立方体单元中的特征线抽取

Figure 2 Extraction of the feature line segment in a cell. (a) The isosurface $S_{f}$; (b) the isosurface $S_{g}$; (c) intersection of $S_{f}$ and $S_{g}$; (d) the feature line segment in the cell

\section{GPU 算法实现}

本节阐述本文算法在 GPU 中的具体实现. 其中, 第 4.1 小节介绍特征线的并行抽取算法 (实时第 3 步); 第 4.2 小节介绍可见性判定, 包括深度图生成 (实时第 2 步) 和可见性判定 (实时第 4 步); 第 4.3 小节介绍预处理中的数据压缩; 第 4.4 小节介绍桶分类方法 (预处理及实时第 1 步).

\section{1 特征线抽取}

特征线抽取是本文算法的一个关键步骤. 我们提出一种基于 GPU 的特征线抽取算法, 并行计算 每个立方体单元中的特征线片段. 如图 2 所示, 对于每个立方体单元, 我们将它内部的特征线片段用 一条直线段来拟合. 该特征线段的两个端点分别位于立方体的两个面上. 为此, 我们分别在立方体的 6 个面上计算特征线与该面是否存在交点. 如果在两个不同的面上存在交点,则将其输出为一条直线段.

每个立方体单元中的特征线段抽取流程如下:

(1) 计算 8 个顶点体素的三维坐标 $\boldsymbol{v}_{i}$, 采样体素值, 计算函数值 $f\left(\boldsymbol{v}_{i}\right)$ 与 $g\left(\boldsymbol{v}_{i}\right)$;

(2) 对于立方体单元的 6 个面 $\mathcal{F}_{i}$, 分别计算面 $\mathcal{F}_{i}$ 与特征线是否存在交点 $\boldsymbol{A}$, 即满足 $\boldsymbol{A} \in \mathcal{F}_{i}$ 且 $f(\boldsymbol{A})=g(\boldsymbol{A})=0$.

(3) 如果在两个面上存在特征线交点, 则将其作为顶点输出该立方体单元中的特征线段; 否则该立 方体单元没有特征线段. 


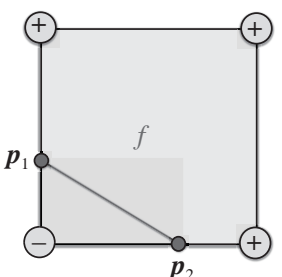

(a)

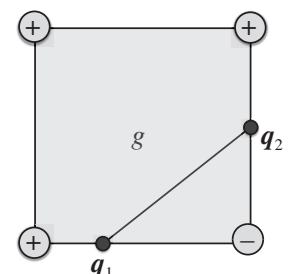

(b)

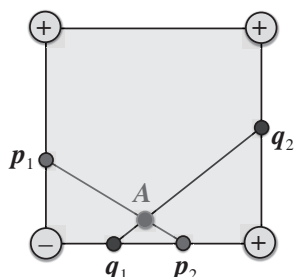

(c)

图 3 立方体表面的特征线顶点计算

Figure 3 Computing the end point of feature line on a face of a cell. (a) Compute the intersection of $\mathcal{F}_{i}$ and $S_{f}$, denoted by $\boldsymbol{p}_{1} \boldsymbol{p}_{2}$; (b) compute the intersection of $\mathcal{F}_{i}$ and $S_{g}$, denoted by $\boldsymbol{q}_{1} \boldsymbol{q}_{2} ;$ (c) compute the intersection point $\boldsymbol{A}$ of $\boldsymbol{p}_{1} \boldsymbol{p}_{2}$ and $\boldsymbol{q}_{1} \boldsymbol{q}_{2}$

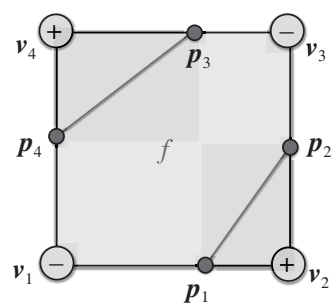

(a)

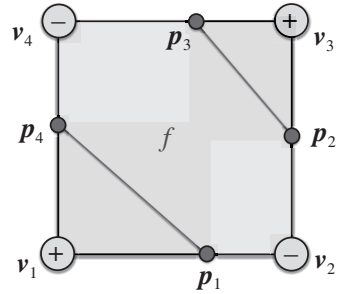

(b)

图 4 面 $\mathcal{F}_{i}$ 与等值面 $S_{f}$ 存在两条交线的情况

Figure 4 The special case when two intersection line segments exist. (a) The intersection is $\boldsymbol{p}_{1} \boldsymbol{p}_{2}, \boldsymbol{p}_{3} \boldsymbol{p}_{4}$; (b) the intersection is $\boldsymbol{p}_{1} \boldsymbol{p}_{4}, \boldsymbol{p}_{2} \boldsymbol{p}_{3}$

以上第 2 步计算面 $\mathcal{F}_{i}$ 与特征线交点的方法如图 3 所示:

(1) 计算 $\mathcal{F}_{i}$ 与等值面 $S_{f}$ 的交线 $\boldsymbol{p}_{1} \boldsymbol{p}_{2}$;

(2) 计算 $\mathcal{F}_{i}$ 与等值面 $S_{g}$ 的交线 $\boldsymbol{q}_{1} \boldsymbol{q}_{2}$;

(3) 计算两条交线 $\boldsymbol{p}_{1} \boldsymbol{p}_{2}$ 与 $\boldsymbol{q}_{1} \boldsymbol{q}_{2}$ 的交点 $\boldsymbol{A}$;

(4) 如果 $\mathcal{F}_{i}$ 与等值面 $S_{f}$ 或 $S_{g}$ 没有交线 (即 $\boldsymbol{p}_{1} \boldsymbol{p}_{2}$ 或 $\boldsymbol{q}_{1} \boldsymbol{q}_{2}$ 之一不存在), 或者两条交线没有交点, 都说明面 $\mathcal{F}_{i}$ 上不存在特征线端点.

其中, $\mathcal{F}_{i}$ 与等值面 $S_{f}$ 的交线 $\boldsymbol{p}_{1} \boldsymbol{p}_{2}$ 通过以下方法计算. 记 $\mathcal{F}_{i}$ 的顶点为 $\boldsymbol{v}_{1}, \boldsymbol{v}_{2}, \boldsymbol{v}_{3}, \boldsymbol{v}_{4}$, 函数值为 $f_{1}, f_{2}, f_{3}, f_{4}$, 若 $f_{1}$ 与 $f_{2}$ 的符号不同, 则说明边 $\boldsymbol{v}_{1} \boldsymbol{v}_{2}$ 上存在一个函数 $f$ 的零值点 $\boldsymbol{p}_{1}$, 通过线性插值 计算: $\boldsymbol{p}_{1}=(1-\alpha) \boldsymbol{v}_{1}+\alpha \boldsymbol{v}_{2}, \alpha=f_{1} /\left(f_{1}-f_{2}\right)$.

根据 4 个顶点值 $f_{i}$ 的符号, 共存在以下 3 种情况:

(1) 所有顶点值 $f_{i}$ 符号相同 (全正或全负), 不存在交线;

(2) 在两条边上存在函数 $f$ 的零值点 (记为 $\boldsymbol{p}_{1}$ 与 $\boldsymbol{p}_{2}$ ), 则存在一条交线 $\boldsymbol{p}_{1} \boldsymbol{p}_{2}$;

(3) 在四条边上都存在函数 $f$ 的零值点 (记为 $\boldsymbol{p}_{1}, \boldsymbol{p}_{2}, \boldsymbol{p}_{3}, \boldsymbol{p}_{4}$ ). 如图 4 所示, 分为两种情况. 我们 通过 $\boldsymbol{v}_{1}$ 点的值 $f_{1}$ 来区分两种情况.

在最极端的情况下, 面 $\mathcal{F}_{i}$ 与等值面 $S_{f}, S_{g}$ 分别有两条交线, 最多可产生 4 个交点满足 $f=g=0$. 这样的情况在我们的实验中几乎没有发生过. 在实现中, 对于一个面存在多个交点的情况, 我们计算 它们的平均值作为该面唯一的特征线顶点. 我们在 GPU 的几何处理器 (geometry shader) 中实现以上 介绍的特征线抽取算法. 所有体素的数据 (体素值、法向等) 通过压缩编码的方式保存在二维纹理中 (见第 4.3 小节). 对于每一个立方体单元我们使用一个独立的线程计算 8 个点体素的函数值 $f\left(\boldsymbol{v}_{i}\right)$ 与 


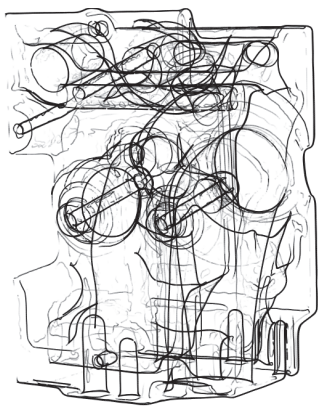

(a)

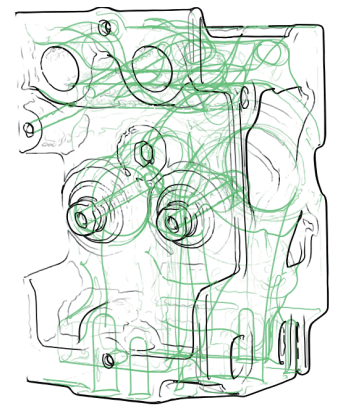

(b)

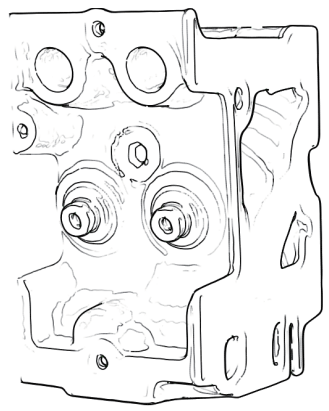

(c)

图 5 使用可见性信息表现空间结构

Figure 5 The importance of visibility in conveying the inner structure. (a) No visibility; (b) distinguish visible/invisible lines with different colors; (c) remove invisible lines

$g\left(\boldsymbol{v}_{i}\right)$, 在 6 个面上分别计算是否与特征线存在交点, 统计交点的数量. 如果在两个不同的面上找到交 点, 则以它们为端点输出一个 OpenGL 的线段单元.

讨论: 我们提出的抽取立方体单元特征线的方法事实上是移动线段法的一种简化. 首先, 我们不 考虑特征线的方向, 也就是说, 我们的方法不能区分 “正面” 及 “反面” 的特征线. 对于线绘制而言, 我 们不需要做出这样的区分, 而是通过下一节介绍的方法区分 “可见” 与 “不可见” 的特征线. 其次, 我 们的方法在一个立方体单元中最多只输出一条特征线段. 而事实上, 在一个立方体中可能有超过两个 面与特征线相交. 在这样的情况下, 我们的方法只能抽取出部分的特征线段. 但是, 在我们的实践中这 样的情况出现得很少. 特别是我们针对的模型都具有较高的分辨率, 导致每个体素在屏幕上的投影面 积很小. 因此, 实际生产的线绘制结果与正确结果的差异微乎其微 (见第 5 节实验结果). 在运行效率 上, 由于我们的算法不需要任何查找表操作, 能有效地利用 GPU 的计算性能, 因此速度比 CPU 上运 行的移动立方体算法能快至两个数量级.

\section{2 可见性判断}

可见性是体模型线绘制算法中需要解决的一个关键性问题. 与网格模型相比, 体模型具有复杂的 内部空间结构 (即使是单个等值对应的等值面也具有多层结构). 由于特征线的稀疏特性, 可以将多层 表面的特征线叠加在一起从而表现体模型的空间结构. 但是, 如图 5(a) 所示, 在绘制时如果不考虑特 征线的可见性, 绘制结果会显得杂乱而难以理解; 相反, 如果将不可见的特征线使用不同的属性 (如颜 色) 进行区分 (图 5(b)), 或者直接去除不可见的特征线 (图 5(c)), 能更清晰的表现体模型的空间结构.

我们提出一种基于 splatting 深度图的可见性判定方法: 首先, 我们找出所有与当前等值面 $S_{f}$ 相 交的立方体单元. 一个立方体单元 $\mathcal{C}$ 与 $S_{f}$ 相交当且仅当 $f_{\min } \leqslant f_{0} \leqslant f_{\max }, f_{\min }, f_{\max }$ 表示 $\mathcal{C}$ 的 8 个顶点体素值的最小值与最大值, $f_{0}$ 是当前等值面值. 其次, 对于每个与 $S_{f}$ 相交的立方体单元, 我们 以其中心为原点绘制一个 splat 圆盘, splat 的法向为周围 8 个体素法向的平均值, 半径为 2 倍体素 半径. Splatting 的结果是生成当前可见等值面的深度图 (见图 1), 基于该深度图只需在几何处理器 (geometry shader) 中进行简单的深度比较就可以确定每个立方体单元中特征线段的可见性.

由于相邻的 splat 圆盘之间互相重叠, 交叉, 使用 splatting 方法生成的深度图并不完全精确, 从而 造成如图 6 (a) 所示的深度冲突的问题, 将一些可见的特征线判定为不可见, 或者将不可见的特征线 判定为可见. 为了避免这样的问题, 我们提出一种自适应的深度偏移方法, 将每个 splat 沿着视线方向 


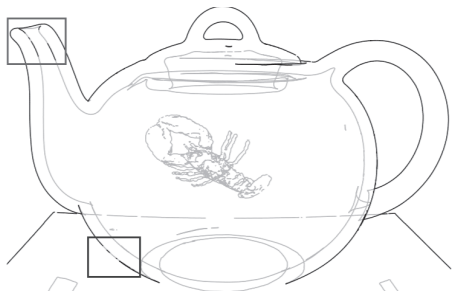

(a)
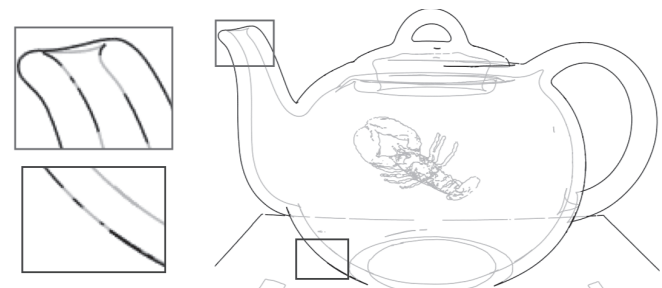

(b)

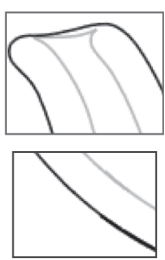

图 6 固定深度偏移和自适应深度偏移的效果差异

Figure 6 Constant depth offset vs. adaptive depth offset. (a) Artifacts generated by constant depth offset; (b) adaptive depth offset leads to correct result

偏移一定的距离 $d: d=r \cdot\left(\sqrt{1-D^{2}} / D \cdot a+b, d_{\max }\right), D=|\boldsymbol{n} \cdot \boldsymbol{v}|$, 以上公式的直观含义是, 对于正对于 视线方向的 splat 采用较小的偏移, 对于与视线方向夹角较大 (接近于侧影轮廓线) 的 splat 采用较大 的偏移. 这实际上是对 OpenGL 多边形偏移的一种模拟. 在实验中, 我们设置 $a=5, b=2, d_{\max }=10$, 对所有测试的模型都能取得较好的结果. 图 6 比较了采用固定深度偏移和自适应深度偏移的差别.

讨论: 已有工作 ${ }^{[10,11]}$ 通过光线投射的方法判断可见性: 对特征线段的每个顶点, 向视线方向投射 一条射线, 用 $3 \mathrm{D} \mathrm{DDA}$ 算法检查该射线是否与当前等值面 $S_{f}$ 相交. 若相交, 则说明该顶点不可见, 否 则可见. 这样的方法可以在 GPU 中并行实现, 但是 $3 \mathrm{D} \mathrm{DDA}$ 算法需要对体数据进行大量的遍历, 在 GPU 中实现时归结为大量的纹理采样, 因此效率很低. 我们的方法只需对所有与当前等值面 $S_{f}$ 相交 的立方体单元遍历一次. 实验结果表明, 我们的方法在效率上比 (GPU 上实现的) 光线投射法要快 10 倍左右. 在效果方面, 在采用自适应深度偏移的情况下, 两种方法都能得到几乎正确的结果.

\section{3 数据压缩}

与网格模型相比, 体模型线绘制算法需要解决的另一困难是体模型往往具有很大的数据量, 因此 必须要考虑如何实现高效的数据压缩与解压缩算法. 例如, 一个 $256^{3}$ 分辨率的普通大小的体模型, 对 于每个体素至少需要存储它的三维坐标, 体素值与法向, 在不压缩的情况下每个体素需要占用 24 个字 节, 总的存储消耗达到 $0.4 \mathrm{~GB}$, 还不考虑其他存储消耗 (如深度图、桶分类的索引表等). 再者, 不同 的特征线还需要对每个体素存储额外的信息 (如拉普拉斯线需要存储预计算的法向拉普拉斯, 是一个 三维向量). 对于更高分辨率的体模型, 根本无法将所有数据无压缩地装载到高速的 GPU 显存中. 如 果只加载部分数据又会明显地降低实时绘制的效率.

我们提出数据剔除和数据编码两种方法降低存储开销, 增强体模型线绘制算法的实用性.

数据剔除 我们注意到在一般的体模型中一个常见的现象是, 绝大多数的体素具有极端 (非常大 或非常小) 的体素值, 与这些体素相交的等值面通常并没有任何意义, 因此在线绘制中完全可以将它 们剔除掉而不予考虑. 为此, 我们在装载模型的时候让用户设置两个阈值 $\varepsilon_{\min }$ 与 $\varepsilon_{\max }$, 对于所有体素 值小于 $\varepsilon_{\min }$ 的体素, 将其用唯一一个体素数据表示: 值统一记为 $\varepsilon_{\min }$, 法向统一记为 $(0,0,1)^{\mathrm{T}}$. 对于 所有体素值大于 $\varepsilon_{\max }$ 的体素同样处理. 这样, 我们将体素的数量由 $n$ 降为 $m+2$, 其中 $n$ 是体模型原 有体素数量, $m$ 是体素值位于 $\varepsilon_{\min }$ 与 $\varepsilon_{\text {max }}$ 之间的有效体素数量. 通常, $m \ll n$. 例如, 对于 “波士顿 茶壸” 模型, 当设置 $\varepsilon_{\text {min }}=0.01, \varepsilon_{\text {max }}=0.99$ 的时候 (体素值归一化到 $[0,1]$ ), $n$ 为 1200 万而 $m$ 为 100 万. 在实现中, 我们将个体素数据保存到多个二维纹理中, 用一个额外的三维纹理记录每个体素在二 维纹理中对应的纹理坐标. 值得注意的是, 本节介绍的数据剔除方法要求的一个前提条件是体模型的 
极大或极小体素值可以直接剔除. 尽管本文测试的所有体模型均满足这一条件, 但是不排除存在一些 特殊的体模型, 其任何体素值均需要保留. 对于这样的模型, 我们不进行数据剔除, 这对于其他的算法 流程没有影响.

数据编码 对于未被剔除的体素, 我们通过特定的编码格式对其坐标、法向等数据进行压缩 存储:

- 三维坐标 $(x, y, z)$ : 使用 GL_UNSIGNED_INT_2_10_10_10_REV 格式存储在 OpenGL 顶点缓冲 对象 $(\mathrm{VBO})$ 中, 每个坐标分量用 10 位表示, 结合 alpha 分量, 最大可以支持 $2048 \times 2048 \times 1024$ 的分 辨率;

- 法向 $\left(n_{x}, n_{y}, n_{z}\right)$ : 使用 32 位无符号整数 GL_R32UI 格式编码, 每个分量用 10 位表示, 在 GPU 渲染器 (shader) 中实现法向的解码计算;

-二维纹理坐标 $(u, v)$ : 使用 32 位无符号整数 GL_R32UI 格式编码, 每个分量用 16 位表示, 在 GPU 渲染器 (shader) 中实现法向的解码计算. 最多可以存储约 43 亿个体素的纹理坐标.

通过数据编码我们显著地降低了存储开销. 其中, 对于三维体素坐标及二维纹理坐标的压缩是无 损的, 而对于法向的量化虽然是有损压缩, 但是从实验结果中看与不压缩的结果几乎没有任何视觉上 的差异. 在运行效率方面, 尽管我们需要在 shader 中通过一定的位运算和算术运算来对压缩数据进行 解码, 但是由于带宽消耗的减少, 实时绘制速度反而比不压缩的情况快 $2 \sim 4$ 倍.

\section{4 体数据的桶分类}

在大多数情况下, 特征线的分布是非常稀疏的, 即绝大部分的立方体单元中不存在特征线段, 如何 快速地将不存在特征线的立方体单元快速剔除对于实时绘制效率有相当大的影响. 正是因为如此, 已 有工作 ${ }^{[10,11]}$ 才采用随机种子跟踪的方式, 根据随机生成的种子向周围跟踪出整条特征线.

我们提出通过对体数据进行桶分类的方法快速剔除大部分不相关的立方体单元. 我们将区间 $\left[\varepsilon_{\min }, \varepsilon_{\max }\right]$ 均匀地分为 $k$ 个子区间. 对每个子区间 $\left[\varepsilon_{1}, \varepsilon_{2}\right)$, 我们将与该子区间对应的等值面集合 可能相交的所有体素保存在一个列表 $\mathcal{L}$ 中. 设 $\mathcal{C}$ 是任一立方体单元, 其 8 个顶点体素的最小最大值为 $f_{\text {min }}, f_{\text {max }}$, 则 $\mathcal{C} \in \mathcal{L}$ 当且仅当 $\left[\varepsilon_{1}, \varepsilon_{2}\right) \cap\left[f_{\text {min }}, f_{\text {max }}\right] \neq \emptyset$. 在实时绘制的时候, 对于用户当前指定的等值 面值 $f_{0}$, 我们首先找出 $f_{0}$ 所对应的子区间及列表 $\mathcal{L}_{0}$. 根据构造方式, 显然只有属于列表 $\mathcal{L}_{0}$ 的立方 体单元才有可能与等值面 $S_{f}$ 相交, 因此也只有属于列表 $\mathcal{L}_{0}$ 的立方体单元才有可能存在特征线段. 因 此, 我们在实时绘制的各个阶段 (splatting 深度图、等值线抽取、可见性判定), 都只对属于 $\mathcal{L}_{0}$ 的立方 体单元进行处理. 通过这样的方式, 有效地避免了对绝大多数不相关体素的处理, 显著地提高了实时 绘制速度. 在实现中, 我们将每个子区间对应的体素列表保存为一个 OpenGL 顶点缓冲对象 (VBO). 其中, 对每个体素我们按照上一节介绍的方法将其三维坐标 $(x, y, z)$ 编码为一个 32 位的整数进行 存储.

桶分类的一个副作用是会在一定程度上增大存储开销, 这是由于当一个立方体单元的 8 个顶点体 素值相差较大时, 该立方体单元会被多个桶所记录. 但根据我们的实验观察, 对于我们的测试数据, 在 大部分的立方体单元中, 8 个顶点的数值差别不会太大, 因此多次记录所产生的额外开销并不太大.

\section{5 实验结果}

我们在一台配置英特尔 i3 CPU, 4 GB 内存, 英伟达 $650 \mathrm{Ti}$ 显卡的普通 PC 上实现本文算法并对 1392 


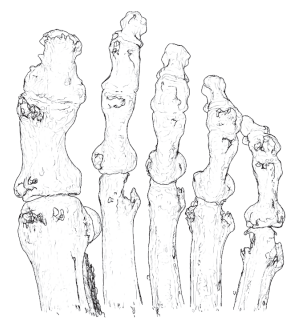

(a)

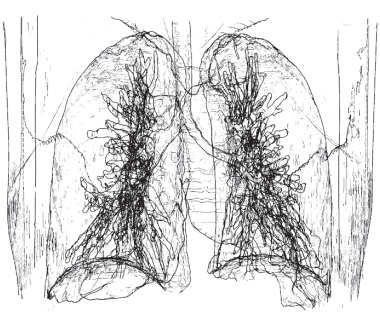

(b)

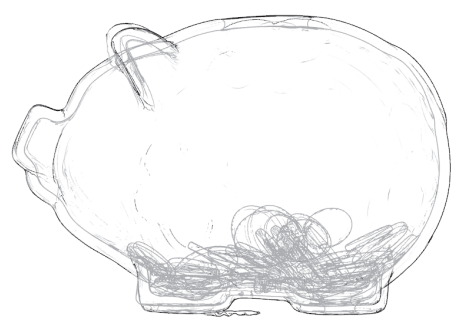

(c)

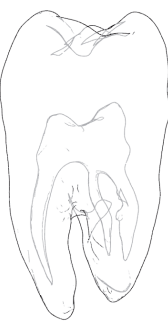

(d)

图 7 本文算法生成的部分线绘制结果

Figure 7 Results generated using our method. (a) Foot (36 fps); (b) chest (45 fps); (c) piggy (38 fps); (d) tooth (334 fps)

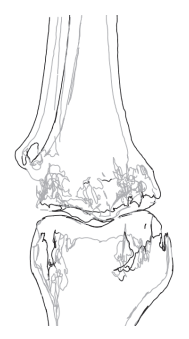

(a)

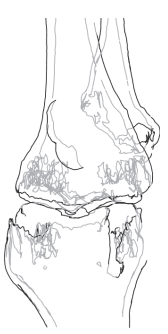

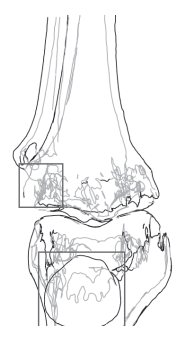

(b)

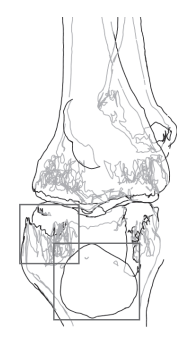

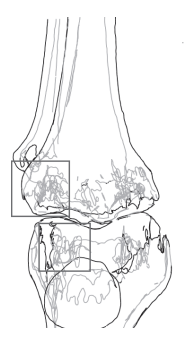

(c)

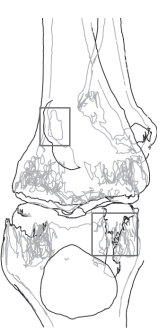

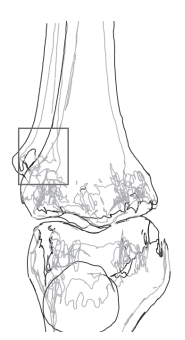

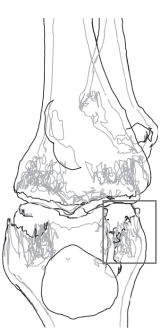

(d)

图 8 随机种子跟踪算法的时空不连续现象

Figure 8 The temporal-coherence problem of the seed-and-traversal approach. (a)-(d) showing the 1st, 2nd, 5th and 10th frame in the continuous update, respectively. The boxes highlight the differences between the current and previous frames. Our method does not suffer from such problem

多个常见体模型进行了测试. 图 7 展示了本文算法生成的部分线绘制结果.

\section{1 算法效果对比}

与已有 $\mathrm{CPU}$ 算法相比, 本文算法在效果上的一大改进在于我们有效地解决了线绘制的时空不连 续现象. 已有 CPU 算法通过跟踪随机产生的种子点得到特征线的几何信息. 由于种子点是随机产生 的, 因而无法确保抽取出所有的特征线. 为此, 在绘制时需要通过多次迭代, 在原有结果基础上添加新 的种子点, 从而不断地更新线绘制结果. 图 8 展示了一个多次迭代更新的实例. 由于相邻帧之间 (如图 $8(a),(b))$ 的线绘制结果可能存在较大差异, 因而产生了闪炼的现象. 本文算法保证每一帧抽取所有的 特征线, 因而不存在这样的时空不连续问题.

为了效率考虑, 本文算法在每个立方体单元中最多只产生一条特征线片段. 这是一种近似的做法, 因为从理论上讲每个立方体单元中可能存在多条特征线片段. 但是, 在我们的实验中发现, 一个立方 体单元中存在多条特征线片段的情况非常少见, 使用本文的近似做法生成的线绘制结果与完整结果在 视觉上的差异非常小. 图 9 给出了一个差异对比的实例, 其中, 图 9(b) 用红色表示出具有差异的所有 特征线片段 (几乎不可见).

本文算法事实上是一个统一的体模型线绘制框架, 通过定义不同的特征函数 $g(x, y, z)$, 可以生 成不同的特征线绘制效果. 除了轮廓线以外，我们还实现了拉普拉斯线的绘制, 其特征函数定义为 $g^{\prime}(x, y, z)=\triangle \boldsymbol{n}(x, y, z) \cdot \boldsymbol{v}(x, y, z)$, 对每个体素 $(x, y, z), \triangle \boldsymbol{n}$ 是按照文献 [11] 的方法预计算的法向 拉普拉斯, $\boldsymbol{v}$ 是视线方向. 图 10 展示了龙虾模型的不同特征线绘制效果. 


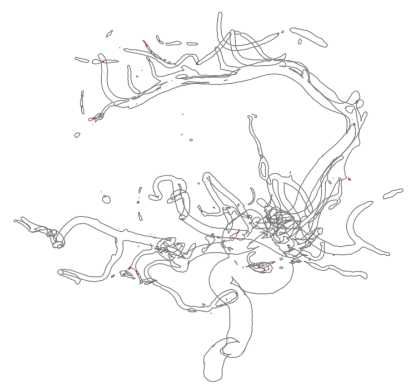

(a) (b)

图 9 本文算法与精确结果的差异

Figure 9 Comparison of our result and ground truth. (a) Difference showing in red; (b) showing the difference alone

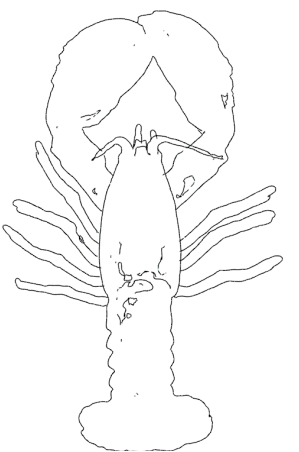

(a)

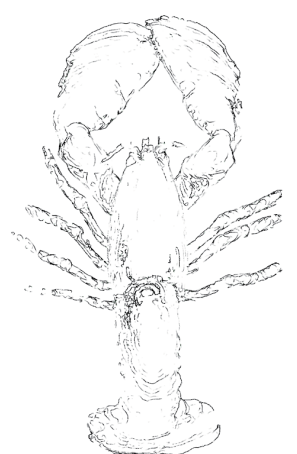

(b)

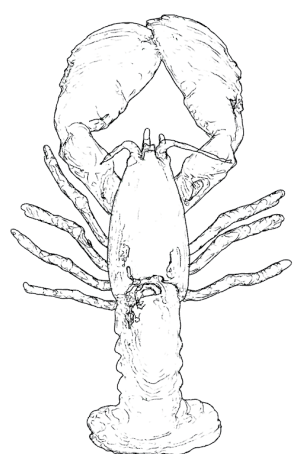

(c)

图 10 不同的特征线绘制结果

Figure 10 Results of different feature lines. (a) contours; (b) Laplacian lines; (c) contours + Laplacian lines

\section{2 算法效率对比}

如图 1 所示, 本文算法分为预处理和实时计算两个阶段. 其中, 预处理阶段完成体数据的桶分类 及各种数据的压缩编码; 实时计算包括特征线的抽取和可见性判定等. 预处理只需在读取体数据后进 行一次. 预处理的时间与模型大小成正比, 对于本文列举的体模型, 预处理的时间在几秒到几十秒之 间, 远小于从硬盘上读取模型数据所花费时间.

表 1 对比了本文算法与已有的基于随机种子跟踪算法 [11] 的实时绘制效率. 所有时间数据均在我 们的测试平台上统计得到. 本文算法的实时绘制速度比 CPU 算法 [11] 快 5 25 倍. 其中, 对于拉普拉 斯线绘制本文算法的加速作用更加明显. 这是由于随机种子跟踪算法的效率与特征线的密集程度有 关, 拉普拉斯线与轮廓线相比相对比较密集, 因而随机种子跟踪法的效率也较低. 本文算法不存在这 样的限制, 对于轮廓线和拉普拉斯线都能达到较高的绘制速度.

表 2 展示了本文采用的桶分类加速算法的作用. 如表 2 所示, 采用桶分类的方法可以快速地剔 除绝大多数 ( $95 \%$ 以上) 不存在特征线片段的立方体单元, 从而显著地提高实时绘制速度. 当然, 加速 性能并不能达到与剔除数量成正比. 这是由于被剔除的立方体单元都是与当前等值面 $S_{f}$ 不相交的, 4.1 小节介绍的等值线抽取可以快速判断这种情况, 相对于存在特征线片段的立方体单元, 计算量较 小. 在实验中, 我们发现使用桶分类大致可以得到 5 10 倍的速度提升. 
表 1 本文算法与基于随机种子跟踪的 CPU 算法的实时绘制效率对比 ${ }^{\text {a) }}$

Table 1 Timing statistics of previous CPU-based seed-and-traversal approach and our GPU-based algorithm

\begin{tabular}{ccccccc}
\hline Model & Resolution & \# Voxels (M) & CPU-C (fps) & GPU-C (fps) & CPU-(C+LL) (fps) & GPU-(C+LL) (fps) \\
\hline Lobster & $301 \times 324 \times 56$ & 5.5 & 26 & 414 & 9 & 195 \\
Male & $128 \times 256 \times 256$ & 8.4 & 23 & 214 & 8 & 151 \\
Tooth & $256 \times 256 \times 161$ & 10.6 & 31 & 530 & 13 & 334 \\
Teapot & $256 \times 256 \times 178$ & 12 & 26 & 180 & 6 & 97 \\
Bonzai & $256 \times 256 \times 256$ & 17 & 8 & 113 & 2.5 & 56 \\
Knee & $379 \times 229 \times 305$ & 26 & 11 & 91 & 2.4 & 43 \\
Fish & $512 \times 512 \times 134$ & 34 & 7.6 & 43 & 2.1 & 24 \\
Chest & $384 \times 384 \times 220$ & 35 & 20 & 92 & 2.6 & 45 \\
\hline
\end{tabular}

a) The 2nd and 3rd columns are the resolution and number of voxels of the model; the 4th and 5th columns show the frame rates of drawing contours with previous CPU algorithm and our GPU algorithm; the 6th and 7th columns show the frame rates of drawing contours and Laplacian lines with previous CPU algorithm and our GPU algorithm.

表 2 体数据桶分类的效果 $\mathrm{b}$ )

Table 2 Effect of the bucket sorting algorithm

\begin{tabular}{cccccc}
\hline Model & \# Voxels $(\mathrm{M})$ & \# In-bucket $(\mathrm{K})$ & Original (fps) & Acceleration (fps) & Acceleration ratio \\
\hline Lobster & 5.5 & 124 & 40 & 195 & 4.8 \\
Male & 8.4 & 350 & 30 & 151 & 4.9 \\
Tooth & 10.6 & 64 & 28 & 334 & 12 \\
Teapot & 12 & 380 & 32 & 180 & 5.6 \\
Knee & 26 & 780 & 13 & 91 & 7 \\
Fish & 34 & 425 & 8 & 43 & 5.2 \\
\hline
\end{tabular}

b) The 2nd column shows the number of total voxels; The 3rd shows the number of voxels in the current bucket; the 4 th and 5 th columns show the frame rates without \& with the bucket sorting algorithm (in fps); and the last column shows the acceleration ratios.

\section{3 数据压缩效果}

表 3 展示了 4.3 小节介绍的数据压缩算法对于一些体模型的压缩效果. 在不使用压缩编码的情况 下, 每个体素需要保存体素值, 法向, 法向拉普拉斯, 共计 28 字节; 使用压缩编码降为 10 字节. 桶分 类的存储量也可以降低为原存储量的 $1 / 3$ 至 $1 / 4$. 另外, 数据剔除能减少 $50 \%$ 至 $90 \%$ 的存储量. 总体 而言, 我们的算法可以在不影响绘制结果和效率的情况下获得 $10 \%$ 至 $30 \%$ 的压缩比.

\section{6 小结}

本文提出一个基于 GPU 的体模型线绘制算法框架. 首先, 我们提出了一种并行的特征线抽取算 法, 可以在几何处理器中并行计算每个立方体单元中的特征线片段. 其次, 我们采用 splatting 的方法 生成深度图以判定特征线的可见性, 通过自适应的深度偏移改进可见性判定效果. 再者, 我们通过剔 除无关数据和压缩编码的方式显著地减少了存储消耗和传输带宽. 本文算法虽然没有采用随机种子跟 踪的加速策略, 但是仍然比已有 CPU 算法快 20 倍, 并且有效地避免了已有算法的时空连续性问题. 
表 3 压缩与非压缩的存储开销对比 ${ }^{\mathrm{c})}$

Table 3 Effect of the compression algorithm

\begin{tabular}{cccc}
\hline Model & Original storage $(\mathrm{MB})$ & Compressed storage (MB) & Compression ratio (\%) \\
\hline Lobster & 260 & 78 & 30 \\
Male & 400 & 121 & 30 \\
Tooth & 506 & 142 & 28 \\
Teapot & 560 & 71 & 13 \\
Knee & 1270 & 172 & 13 \\
Fish & 1610 & 282 & 18 \\
\hline
\end{tabular}

c) The 2nd and 3rd columns are the storage costs of the video memory without \& with compression; the last column shows the compression ratios.

我们的现有算法仅能得到离散的特征线片段.在未来工作中, 我们将研究如何在 GPU 中将特征 线片段连接成连续的特征线. 其次, 本文提出的自适应深度偏移方法尽管能在大部分时候得到令人满 意的可见性检测结果, 但无法保证在任何情况下都得到正确结果. 事实上, 如何得到精确的特征线可见 性, 目前还没有理想的解决方法. 另外, 我们将研究如何在本文框架下实现更多种类的特征线绘制.

\section{参考文献}

1 Raskar R, Tan K H, Feris R, et al. Non-photorealistic camera: depth edge detection and stylized rendering using multi-flash imaging. ACM Trans Graph, 2004, 23: 679-688

2 Kolomenkin M, Shimshoni I, Tal A. Demarcating curves for shape illustration. ACM Trans Graph, 2008: 27: 157

3 DeCarlo D, Finkelstein A, Rusinkiewicz S, et al. Suggestive contours for conveying shape. ACM Trans Graph, 2003, 22: $848-855$

4 Ohtake Y, Belyaev A, Seidel H P. Ridge-valley lines on meshes via implicit surface fitting. ACM Trans Graph, 2004, 23: $609-612$

5 Judd T, Durand F, Adelson E. Apparent ridges for line drawing. ACM Trans Graph, 2007, 26: 19

6 Kang H, Lee S, Chui C K. Coherent line drawing. In: Proceedings of the 5th International Symposium on NonPhotorealistic Animation and Rendering, New York, 2007. 43-50

7 Lee Y, Markosian L, Lee S, et al. Line drawings via abstracted shading. ACM Trans Graph, 2007, 26: 18

8 Zhao H L, Jin X G, Shen J B, et al. Smooth line drawing on graphics hardware. Chinese J Comput, 2009, 32 [赵汉 理, 金小刚, 沈建冰, 等. 基于图形硬件的光滑线条绘制. 计算机学报, 2009, 32]

9 Kolomenkin M, Shimshoni I, Tal A. On edge detection on surfaces. In: Proceedings of IEEE Conference on Computer Vision and Pattern Recognition, Miami, 2009. 2767-2774

10 Burns M, Klawe J, Rusinkiewicz S, et al. Line drawings from volume data. ACM Trans Graph, 2005, 24: 512-518

11 Zhang L, He Y, Xia J, et al. Real-time shape illustration using Laplacian lines. IEEE Trans Vis Comput Graph, 2011, 17: $993-1006$

12 Xie X, He Y, Tian F, et al. An effective illustrative visualization framework based on photic extremum lines (PELs). IEEE Trans Vis Comput Graph, 2007, 13: 1328-1335

13 Thirion J P, Gourdon A. The 3D marching lines algorithm. Graph Mod Image Process, 1996, 58: 503-509

14 Zhang L, He Y, Seah H S. Real-time computation of photic extremum lines (PELs). Vis Comput, 2010, 26: 399-407

15 Kim Y, Yu J, Yu X, et al. Line-art illustration of dynamic and specular surfaces. ACM Trans Graph, 2008, 27: 156

16 Griffin W, Wang Y, Berrios D, et al. GPU curvature estimation on deformable meshes. In: Proceedings of Symposium on Interactive 3D Graphics and Games, New York, 2011. 159-166

17 Griffin W, Wang Y, Berrios D, et al. Real-time GPU surface curvature estimation on deforming meshes and volumetric data sets. IEEE Trans Vis Comput Graph, 2012, 18: 1603-1613

18 Zhang L, Sun Q, He Y. Splatting lines: an efficient method for illustrating 3D surfaces and volumes. In: Proceedings 
of the 18th Meeting of the ACM SIGGRAPH Symposium on Interactive 3D Graphics and Games, New York, 2014.

$135-142$

\title{
GPU-based expressive line drawing algorithm for volumes
}

\author{
ZHANG Long $^{1 *}$, WANG YiGang ${ }^{1} \&$ WU XiangYang ${ }^{2}$ \\ 1 School of Media and Design, Hangzhou Dianzi University, Hangzhou 310018, China; \\ 2 School of Computer Science, Hangzhou Dianzi University, Hangzhou 310018, China \\ *E-mail: hnex.zhang@gmail.com
}

\begin{abstract}
Existing line drawing algorithms for volumes extract feature lines by tracing randomly selected seeds, which is known to have severe temporal coherence problems. We propose a GPU-based line drawing algorithm framework for volume data. The algorithm extracts all feature lines in every frame, eliminating the discontinuity artifacts in successive frames. Firstly, we propose a parallel algorithm to extract feature line segments in each cell in the geometry shader. To determine the visibility of feature lines, we use splatting to render a depth map for each frame, achieving much higher performance than the ray-casting method adopted in previous approaches. Correct visibility is obtained by using adaptive depth offsets. To store the large volume data in the high-speed video memory, we propose efficient data pruning and encoding schemes, which remarkably reduce the memory consumption and bandwidth, while maintaining the line drawing quality. Experimental results show that, our method can render high quality line drawings with temporal coherence, with a rendering speed that is an order of magnitude faster than previous CPU-based methods using seed-and-traversal.
\end{abstract}

Keywords rendering, volume rendering, feature extraction, illustration, acceleration

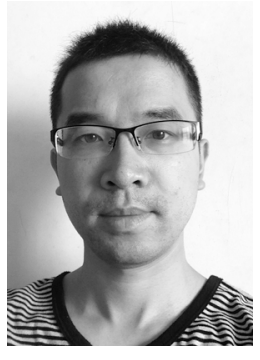

ZHANG Long was born in 1981. He received the Ph.D. degree in Applied Mathematics from the Zhejiang University, Hangzhou city, in 2008. Currently, he is an associate professor at Hangzhou Dianzi University. His research interests include expressive rendering and human-computer interaction.

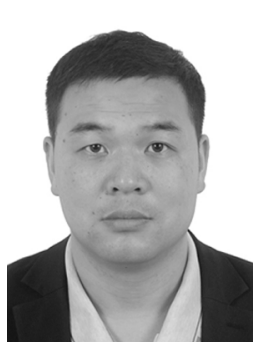

WU XiangYang was born in 1975. He received his Ph.D. degree in Maths from Zhejiang University, Hangzhou China, in 2006. Currently he is an associate professor in Hangzhou Dianzi University. His research interests include realistic rendering, volume data compression and visual analytics.

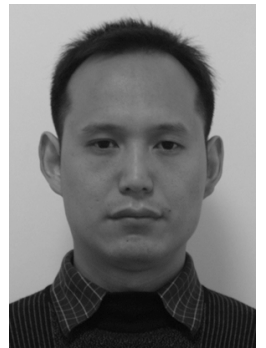

WANG YiGang was born in 1971. He received the Ph.D. degree in Applied Mathematics from Zhejiang University in China, in 1999. Currently, he is a professor in Hangzhou Dianzi University. His research interests include Virtual Reality, Computer Graphics and Image Processing. 